TECHNICAL NOTES AND SHORT PAPERS

\title{
On a Theorem of Piatetsky-Shapiro and Approximation of Multiple Integrals
}

\section{By Seymour Haber and Charles F. Osgood}

Abstract. Let $f$ be a function of $s$ real variables which is of period 1 in each variable, and let the integral $I$ of $f$ over the unit cube in $s$-space be approximated by

$$
Q(f)=\frac{1}{N} \sum_{r=1}^{N} f(r \mathbf{x})
$$

(where $\mathbf{x}=\mathbf{x}(N)$ is a point in $s$-space). For certain classes of $f^{\prime}$ 's, defined by conditions on their Fourier coefficients, it is shown using methods of N. M. Korobov, that x's can be found for which error bounds of the form $|I(f)-Q(f)|<K(f) N^{-p}$ will be true. However, for the class of all $f$ 's with absolutely convergent Fourier series, it is shown that there are no $\mathbf{x}$ 's for which a bound of the form $|I(f)-Q(f)|$ $=O(F(N))$ will hold, for any $F(N)$ which approaches zero as $N$ goes to infinity.

In his book Number-Theoretic Methods of Approximate Analysis, N. M. Korobov quotes the following result of I. I. Piatetsky-Shapiro [1]:

Theorem. Let $A_{8}$ denote the class of all functions of $s$ real variables that have period 1 in each variable and have an absolutely convergent Fourier series:

$$
f(\mathbf{x})=\sum_{m_{1}, \ldots, m_{s}=-\infty}^{\infty} c(\mathrm{~m}) \exp (\mathbf{x} \cdot \mathbf{m})
$$

(boldface letters denote $s$-tuples of real numbers; $\left.\exp a=e^{2 \pi i a}\right)$. Then for any $f \in A_{8}$ and any positive integer $N$ there is a $\boldsymbol{\theta}$ such that

$$
\left|\int_{0}^{1} \ldots \int_{0}^{1} f(\mathbf{x}) d \mathbf{x}-\frac{1}{N} \sum_{r=1}^{N} f(r \boldsymbol{\theta})\right|<C \frac{\log N}{N}
$$

where $C=C(f)$.

Though Korobov takes up this theorem in connection with methods of approximate evaluation of multiple integrals, the theorem itself does not provide such a method, as $\boldsymbol{\theta}$ depends on $f$. The question then arises whether a $\boldsymbol{\theta}(N)$ exists which will make (2) true for all $f \in A_{8}$. We answer this in the negative; but we do show that there are $\boldsymbol{\theta}$ 's which allow a stronger statement than (2) for some considerable subsets of $A_{s}$.

We will denote the unit cube in $s$-dimensional Euclidean space by $G_{s}$.

THEOREM 1. If $N_{1}, N_{2}, \cdots$ is an increasing sequence of positive integers, $\boldsymbol{\theta}^{(1)}$, $\boldsymbol{\theta}^{(2)}, \cdots$ a sequence of $s$-tuples of real numbers, and $F(n)$ any positive decreasing function such that $F(n) \rightarrow 0$ as $n \rightarrow \infty$, then there is an $f \in A_{s}$ such that

$$
\left|\int_{G_{s}} f-\frac{1}{N_{i}} \sum_{r=1}^{N_{i}} f\left(r \boldsymbol{\theta}^{(i)}\right)\right| / F\left(N_{i}\right)
$$

is unbounded as $i \rightarrow \infty$.

Received May 8, 1968. 
Proof. $A_{s}$ is a Banach space, if, with the expansion (1), we define

$$
\|f\|=\sum_{m_{1}, \ldots, m_{s}=-\infty}^{\infty}|c(\mathbf{m})| .
$$

Define the linear functional $L_{i}, i=1,2, \cdots$, by

$$
L_{i}(f)=\frac{1}{F\left(N_{i}\right)}\left(\int_{G_{s}} f-\frac{1}{N_{i}} \sum_{r=1}^{N_{i}} f\left(r \theta^{(i)}\right)\right) .
$$

If the theorem does not hold, then $\left|L_{i}(f)\right| \leqq C(f), i=1,2, \cdots$ for every $f \in A_{\varepsilon}$, where $C(f)$ is some real number. Then by the Banach-Steinhaus Theorem (see, e.g., [2]) there is a constant $K$ such that

$$
\left|L_{i}(f)\right| \leqq K\|f\|
$$

for all $i$ and all $f \in A_{s}$. But if we choose $\mathrm{m}^{(i)}$, for each $i$, in such a manner that $\mathrm{m}^{(i)} \cdot \boldsymbol{\theta}^{(i)}$ is within $1 / 2 N_{i}{ }^{2}$ of an integer (and the components of $\mathrm{m}^{(i)}$ are not all zero), and set $f_{i}(\mathbf{x})=\exp \left(\mathbf{x} \cdot \mathrm{m}^{(i)}\right)$, then

$$
\left|L_{i}\left(f_{i}\right)\right|=\frac{1}{N_{i} F\left(N_{i}\right)}\left|\sum_{r=1}^{N_{i}} \exp \left(r^{(i)} \cdot m^{(1)}\right)\right| \geqq \frac{1}{2 F\left(N_{i}\right)},
$$

contradicting (4).

If

$$
D=\sum_{m_{1}, \ldots, m_{s}=-\infty}^{\infty} d(\mathrm{~m})
$$

is a convergent ( $s$-tuple) series of positive constants, we shall denote by $A_{s}(D)$ the subset of $A_{s}$ consisting of those functions having expansions (1) satisfying

$$
|c(\mathrm{~m})| \leqq C d(\mathrm{~m}), \quad-\infty<m_{1}, \cdots, m_{\mathrm{s}}<\infty
$$

for some number $C$.

Theorem 2. If $D$ is any convergent s-tuple series of positive numbers, and $N$ is a prime number, then there are integers $a_{1}, a_{2}, \cdots, a_{s}$ between 0 and $N-1$ such that

$$
\left|\int_{G_{s}} f-\frac{1}{N} \sum_{r=1}^{N} f\left(r \frac{a_{1}}{N}, r \frac{a_{2}}{N}, \cdots, r-\frac{a_{s}}{N}\right)\right|<\frac{K(f)}{N}
$$

for all $f \in A_{s}(D)$.

Proof. Using the expansion (1), we see that $\int_{G_{g}} f=c(0, \cdots, 0)$ while

$$
\begin{aligned}
\frac{1}{N} \sum_{r=1}^{N} f\left(\frac{r}{N} a\right) & =\sum_{m_{1}, \ldots, m_{s}=-\infty}^{\infty} c(\mathbf{m})\left(\frac{1}{N} \sum_{r=1}^{N} \exp \left(\frac{r}{N} \mathbf{a} \cdot \mathbf{m}\right)\right) \\
& =c(0, \cdots, 0)+\sum_{m_{1}, \ldots, m_{s}-\infty}^{\infty} c(\mathbf{m}) \delta_{N}(\mathbf{a} \cdot \mathbf{m})
\end{aligned}
$$

where $\delta_{N}(n)$ is 1 if $N$ divides $n$ and is 0 otherwise, and the prime on the sum indicates that the term with $m_{1}=m_{2}=\cdots=m_{s}=0$ is omitted. Therefore

$$
\begin{aligned}
\left|\int_{G_{s}} f-\frac{1}{N} \sum_{r=1}^{N} f\left(\frac{r}{N} \mathbf{a}\right)\right| & \leqq \sum_{m_{1}, \ldots, m_{\mathbf{g}}=-\infty}^{\infty}|c(\mathbf{m})| \delta_{N}(\mathbf{a} \cdot \mathbf{m}) \\
& \leqq C \sum_{m_{1}, \ldots, m_{\mathbf{s}}=-\infty}^{\infty} d(\mathbf{m}) \delta_{N}(\mathbf{a} \cdot \mathbf{m}) .
\end{aligned}
$$


Let us now look at the average, for given $N$ and $\mathbf{m}$, of $\delta_{N}(\mathbf{a} \cdot \mathbf{m})$ over all $s$-tuples a of integers from 0 to $N-1$. Choosing a $j$ such that $m_{j} \neq 0$, we see that for any choice of $a_{1}, \cdots, a_{j-1}, a_{j+1}, \cdots, a_{s}$ there is just one value of $a_{j}$ making $\delta=1$-since $N$ is a prime- and $N-1$ values for which $\delta=0$. Thus for $\rho$ ach $\mathbf{m}$,

$$
\text { av } \delta_{N}(\mathbf{a} \cdot \mathbf{m})=1 / N \text {. }
$$

It follows that

$$
\begin{aligned}
\min _{0 \leqq a_{1}, \ldots, a_{s} \leqq N-1} & \left|\int_{G_{s}} f-\frac{1}{N} \sum_{r=1}^{N} f\left(\frac{r}{N} \mathbf{a}\right)\right| \\
& \leqq \text { av } C \sum_{m_{1}, \ldots, m_{s}=-\infty}^{\prime} d(\mathbf{m}) \delta_{N}(\mathbf{a} \cdot \mathbf{m}) \\
& \leqq \frac{C}{N} \sum_{m_{1}, \ldots, m_{s}=-\infty}^{\infty} d(\mathrm{~m})
\end{aligned}
$$

proving the theorem.

This result has consequences for the numerical integration of functions satisfying certain stronger conditions. If, following Korobov, we set

$$
\bar{m}=\max (|m|, 1), \quad\|\mathrm{m}\|=\bar{m}_{1} \cdot \bar{m}_{2} \cdot \cdots \cdot \bar{m}_{s},
$$

and denote by $E_{s}{ }^{\alpha}$, for $\alpha>1$, the set of functions having an expansion (1) that satisfies $|c(\mathrm{~m})| \leqq C(f)\|\mathrm{m}\|^{-\alpha}$, we have

Corollary 1. For each prime number $P$ and for any positive number $\epsilon$ there are integers $a_{1}, a_{2}, \cdots, a_{s}$ such that for any $f \in E_{s}{ }^{\alpha}$

$$
\left|\int_{G_{s}} f-\frac{1}{P} \sum_{r=1}^{P} f\left(\frac{r}{P} \mathbf{a}\right)\right|<\frac{K(f)}{P^{\alpha-\epsilon}} .
$$

Proof. Set $\beta=\max (\alpha-\epsilon, 1)$ and set

$$
g(\mathbf{x})=\sum_{m_{1}, \ldots, m_{s}=-\infty}^{\infty}\|\mathbf{m}\|^{-\alpha / \beta} \exp (\mathbf{m} \cdot \mathbf{x}) ;
$$

and let a be the $s$-tuple of Theorem 2 . Since $\sum t^{\beta} \leqq\left(\sum t\right)^{\beta}$ whenever $\beta \geqq 1$ and the $t$ 's are nonnegative, the quantity

$$
\sum_{m_{1}, \ldots, m_{s}=-\infty}^{\infty}|c(\mathbf{m})| \delta_{P}(\mathbf{a} \cdot \mathbf{m})
$$

for $f$ is no greater than $C(f)$ times the $\beta$ th power of the same quantity for $g$; and the latter is less than or equal to $K(g) / P$.

Korobov obtains a sharper result than this; where we have $P^{-\alpha+\epsilon}$ in $(9)$ he has $P^{-\alpha} \log ^{\beta} P$ for certain values of $\beta$. If we further restrict the class of functions we obtain a result that does not follow directly from Korobov's theorems:

Let $L_{s}{ }^{\alpha}$, for $\alpha>1$, be the class of all functions having an expansion (1) that satisfies

$$
|c(\mathrm{~m})| \leqq C\left(\|\mathrm{~m}\| \log ^{1+\epsilon}\|\mathrm{m}\|\right)^{-\alpha}
$$

for some $C=C(f)$ and $\epsilon=\epsilon(f)>0$. Then we have, by a proof similar to that of the above corollary, 
Corollary 2. For each prime number $P$ there is a set of integers $a_{1}, \cdots, a_{8}$ such that for any $f \in L_{s}^{\alpha}$

$$
\left|\int_{G_{s}} f-\frac{1}{P} \sum_{r=1}^{P} f\left(\frac{r}{P} \mathbf{a}\right)\right|<\frac{K(f)}{P^{\alpha}} .
$$

National Bureau of Standards

Washington, D. C. 20234

1. N. M. Kововоv, Number-Theoretic Methods of Approximate Analysis, Fizmatgiz, Moscow, 1963, p. 85. (Russian) MR 28 \#716.

2. G. F. Simmons, Introduction to Topology and Modern Analysis, McGraw-Hill, New York, 1965 , p. 239. 